\title{
Nocturnal Deterioration after Ischemic Stroke and Autonomic Dysfunction: Hypothesis and Implications
}

\author{
Iryna Palamarchuk ${ }^{\mathrm{a}, \mathrm{b}}$ Kurt Kimpinski ${ }^{\mathrm{a}}$ b Corbin Lippert $^{\mathrm{a}}$ Vladimir Hachinski ${ }^{\mathrm{a}, \mathrm{b}}$ \\ ${ }^{a}$ Department of Clinical Neurological Sciences, University Hospital, London Health Sciences Centre, and \\ ${ }^{b}$ Schulich School of Medicine and Dentistry, Western University, London, Ont., Canada
}

\section{Key Words}

Ischemic stroke · Early deterioration · Sleep · Autonomic function

\begin{abstract}
Background: A significant number of patients admitted to hospital after acute ischemic stroke deteriorate clinically. Deterioration is generally noted within the first $48 \mathrm{~h}$ after stroke onset. The mechanisms leading to this deterioration are not fully understood. Summary: One potential cause of this deterioration may be altered or impaired autonomic function. We expect the hemodynamic changes regulated by the autonomic nervous system that are dysregulated after stroke to be exaggerated during sleep, resulting in arrhythmia and blood pressure fluctuations in these patients. Such physiological changes could result in worsening the overall outcome of the ischemic stroke patient or in sudden death. Therefore, it is necessary to summarize yet unrelated observations and hypothesize on their individual effects and interactions as they relate to poststroke deterioration. Key Messages: If the hypothesis is correct that dysautonomia occurs to the degree that it affects clinical outcomes negatively, this would have important implications for the prevention of neurological deterioration and sudden death after ischemic stroke.

(c) 2013 S. Karger AG, Basel
\end{abstract}

\section{Introduction}

Clinical deterioration after acute ischemic stroke occurs mostly within the first $48 \mathrm{~h}$ after stroke onset and overnight [1]. Stroke is one of the leading causes of all death globally $[1,2]$, with stroke victims often recovering from the initial ischemia only to decline suddenly and unexpectedly [3]. Potential cerebral mechanisms of early poststroke deterioration are failure of collateralization, clot progression, raised intracranial pressure, seizures, recurrent stroke and hemorrhagic transformation $[4,5]$. Sykora et al. [6] (2009) hypothesized that stroke-related autonomic imbalance may promote secondary brain injury due to local inflammation, hyperglycemia or altered cerebral perfusion due to increased blood pressure (BP) variability and impaired cerebral autoregulation. Hilz et al. [7] (2011) showed that increasing stroke severity is associated with progressive decline of parasympathetic activity, baroreflex sensitivity (BRS), total autonomic cardiac modulation and increased sympathetic drive. Obstructive sleep apnea (OSA) has been independently associated with the first-ever stroke and all-cause mortality [8], and impaired sleep architecture in stroke has been associated with poor clinical outcome $[9,10]$.

The reasons for early deterioration remain unclear, but may be linked to cardiac complications such as ar-

\section{KARGER}

E-Mail karger@karger.com

www.karger.com/ced
(C) 2013 S. Karger AG, Basel

1015-9770/13/0366-0454\$38.00/0
Kurt Kimpinski

Room C7-131, University Hospital, London Health Sciences Centre

339 Windermere Road

London, ON N6A 5A5 (Canada)

E-Mail kkimpin@uwo.ca 
rhythmia and/or BP fluctuations after stroke [7, 11]. Cardiovascular reflexes are strongly influenced by specific sleep phases [12,13], but sleep architecture is altered in stroke $[9,10]$ and the association between sleep structure and cardiovascular events in stroke is not well established.

The pivotal pathophysiological mechanisms of OSA are debated, but some events are linked to cardiovascular modulation: apneic events are associated with reduced BRS during night sleep (whereas it is increased in healthy subjects) [14], and arousals are associated with increased sympathovagal balance [15] and changes of BP [16]. Ding et al. [17] (2004) found that an arousal index was inversely associated with the change in volume in white matter disease; hence, they proposed that arousal response could reflect a protective mechanism against sudden death. We expect the autonomic fluctuations that occur after stroke to be exaggerated during sleep, resulting in arrhythmias and BP fluctuations in these patients. Such mechanisms could result in worsening of clinical outcomes or in sudden death.

\section{Sleep and Acute Stroke}

\section{Sleep Evaluation}

Sleep-related complaints and disorders are common in the general population and significantly affect quality of life $[14,16]$. The specific syndromes that are frequent companions of stroke and relate to risk for stroke and mortality are OSA (50-70\%) and excessive daytime sleepiness (EDS, 20-40\%) [17-19]. As a cerebral bioelectrical phenomenon, sleep is represented by two cycling states that are distinguished by the presence or absence of rapid eye movements (REM) [20]. Non-REM (NREM) sleep is a synchronous brain activity with four stages and is characterized by micro-arousals in cyclic alternating patterns that are transient periods of lightening of sleep depth [21]. The first stage of NREM (drowsy) is represented by mixed frequency activity; the second stage takes about half of total sleep time in adults and is distinguished by sleep spindles $(12-14 \mathrm{~Hz})$; the third and fourth stages are represented by slow-wave activity of $0.5-2 \mathrm{~Hz}$. REM sleep is desynchronized brain activity (usually dreaming) with one stage and is characterized by atonic muscles and sawtooth waves $(2-6 \mathrm{~Hz})$ preceding a burst of REM. A sleep cycle (from the beginning of NREM sleep to the end of REM sleep) usually takes about $1.5 \mathrm{~h}$ [20]. The amount and timing of sleep and sleep stages are determined by several factors. Important

Nocturnal Deterioration after Ischemic

Stroke and Autonomic Dysfunction among these factors are the environment, circadian rhythms and time awake. In the assessment of daytime sleepiness and sleep efficiency, the Multiple Sleep Latency test is helpful, where sleep latency is the time from lights off until onset of each stage of sleep [22]. EEG recording of sleep can be transformed from the time domain into the frequency domain in power spectral analysis, where the EEG quantification demonstrates the distribution of power over frequency in time frame. As sleep is not only a global brain phenomenon but a local one as well, detailed sleep analysis includes examination of cerebral functional connectivity with the help of the coherence technique that measures correlation in the frequency domain [23]. Considering that sleep phases are associated with cardiac modulation by the autonomic nervous system (ANS) $[12,24]$ and that symptomatic presentation of sleep disorders (e.g. EDS) can be combined with pathophysiological (e.g. circadian rhythms) or systemic (e.g. breathing disturbances) manifestations, it is important to evaluate sleep in the context of intersystemic relationships (polysomnography) [25].

Association of Sleep Architecture and Stroke Outcome

In acute stroke, nocturnal brain activity is typically fragmented, and impaired sleep architecture is associated with worse short- and long-term outcome $[6,7,10,12]$. Hachinski et al. [26-28] proposed that the presence of stage 2 of NREM sleep has a prognostic value in stroke outcome. Giubilei et al. [29] (1992) found a link between slow-wave sleep and the amount of stroke volume (positive association). In the 2002 study, Müller et al. [30] showed that slow-wave activity low-ratio NREM/wakefulness negatively correlates with stroke severity. Bassetti and Aldrich [9] (2001) noted that patients with poor short-term outcome had less NREM stage 2 and a significant reduction in well-developed sawtooth waves compared with patients with good short-term outcome. Gottselig et al. [31] (2002) found an association between the power and coherence of sleep spindle frequency activity and long-term stroke outcome. Urakami [32] (2009) showed that reduced spindles were associated with an absence of activation in the ipsilateral hemisphere.

\section{Autonomic Dysfunction and Acute Stroke}

The ANS has been extensively implicated in the triggering of sudden death [33]. The reason for sudden death remains unclear, but may be linked to cardiac complications such as arrhythmias and BP changes in stroke [7, 11]. Davies et al. [34] (1994) found that OSA patients have nondipping BP; thus, they suggested that a sleep- 
related rise in BP may contribute to the excess cardiovascular morbidity and mortality experienced by patients with OSA. Selic et al. [35] (2005) showed that OSA contributes to the overall poststroke increase of systolic and diastolic BP during the first 3 days after stroke onset. The authors confirmed that nondipping BP independently results in greater stroke severity and poor outcome. In acute stroke, systolic BP has increased variability; elevated at stroke onset, it suddenly declines (the higher the initial BP the greater the decline) within a 3- to 10-day period [36, 37].

Sympathetic overactivity plays a key role in the development of essential hypertension [38]. Normotensive OSA patients have higher heart rate and norepinephrine plasma levels at rest during wakefulness and a higher response of arterial BP to head-up tilt compared to controls [39]. Cortelli and Provini [40] (2012) hypothesize that, in OSA, episodic recurrence of sympathetic surges during the night may result in adaptation of the autonomic network (chronic hypertensive state). The causes of sympathetic overactivity are multiple, but all of them have a common pathway through the neural integration of baroreceptor activity [38]. The baroreflex maintains a stable $\mathrm{BP}$ by adjusting the heart rate (vagal component) and total peripheral resistance (sympathetic adrenergic component) $[40,41]$. BRS is reduced in acute stroke patients [37]; importantly, low BRS independently contributes to cardiac mortality [33].

Autonomic dysfunction can emerge from impaired peripheral branch (baroreceptors and sympatho/vagal activity) or from central structures (cerebral cortical and subcortical network that includes the bilateral insular cortex, anterior cingulate gyrus, amygdala and hypothalamus) [40]. Oppenheimer et al. [42] (1992) demonstrated that cardiovascular changes can be provoked by insular stimulation. Abboud et al. [43] (2006) compared insular and noninsular stroke and found that right insular stroke was associated with an increased 2-year mortality (vascular and all-cause).

\section{Evaluation of Autonomic Function}

Greater stroke severity is associated with progressive decline of parasympathetic activity, BRS and total autonomic cardiac modulation, while sympathetic dominance shows an increased drive [7]. The large prospective Autonomic Tone and Reflexes after Myocardial Infarction (ATRAMI) study [33] showed that the prognostic markers of autonomic function that are independent of other vascular risk factors are heart rate variability (standard deviation of normal-to-normal intervals) and BRS.
BRS is a quantitative description of baroreflex gain as a response of arterial BP and heart rate to a sudden increase in BP $[33,37]$. Besides global assessment of BRS, it is reasonable to assess its vagal and adrenergic components separately, as baroreflex is a result of increased vagal activity and decreased sympathetic activity $[44,45]$. The 'gold standard' for the evaluation of adrenergic BRS is a modified Oxford technique [46]; however, this method has limitations as it involves injections of nitroprusside and phenylephrine, and it may alter BRS via inhibition of the central nuclei [47]. The best evaluation of the adrenergic component results from direct recordings of muscle sympathetic nerve activity, but this test is not applicable for routine clinical practice; adrenergic BRS during Valsalva maneuver (VM) is expected to be a more practical measurement [41]. As altered breathing efforts (OSA) are linked to the most reduced BRS during night sleep [14, 45], and independently associated with first-ever stroke and all-cause mortality [8], we consider to evaluate nocturnal spontaneous VM in stroke as a potential prognostic marker (e.g. the more significant the pressure the greater the hemodynamic changes). Certainly, spontaneous VM in poststroke OSA could be lower than one in classical OSA due to less severe apnea-hypopnea index (AHI). In addition, AHI severity may depend upon ANS dysfunction; thus, we suggest evaluating the complex of spontaneous VM and AHI after acute stroke is reasonable.

Given the following factors: (1) BRS is a prognostic and independent autonomic marker of cardiovascular risk [33]; (2) estimation of spontaneous BRS partially includes the gain of the baroreceptors in a closed-loop system; (3) disrupted nocturnal baroreflex gain is potentially linked to the clinical deterioration after acute ischemic stroke $[24,37,38]$; (4) nondipping BP independently results in greater stroke severity and poor outcome [35], and (5) nocturnal cardiac modulation within the first 48 $\mathrm{h}$ after acute ischemic stroke may be responsible for the sudden clinical deterioration [1], nocturnal spontaneous $\mathrm{BRS}$ is a prospective early marker of stroke deterioration.

\section{Potential Role of Autonomic Dysfunction in Nocturnal Deterioration}

The physiological, neurochemical and anatomical aspects of sleep processes are a solid core for cooperation with autonomic regulation in a dynamic and synchronic way (e.g. phasic fluctuations of sympathetic and parasympathetic activity during REM sleep compared with relatively stable parasympathetic predominance in NREM) [40]. There are interesting physiological patterns of car- 
Fig. 1. Normal autonomic repatterning in cardiac modulation regarding sleep phases.

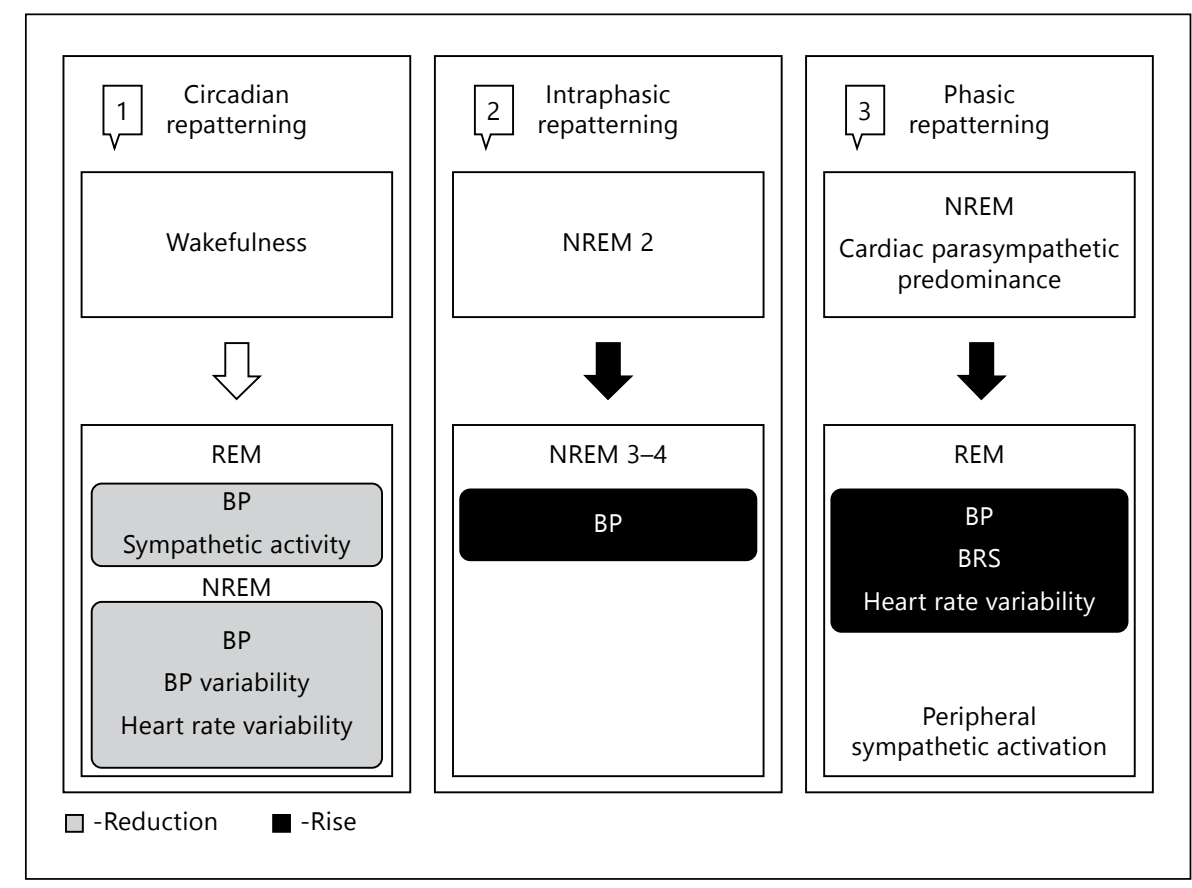

Table 1. Sleep-dependent autonomic modulation

\begin{tabular}{lll}
\hline & NREM sleep & REM sleep \\
\hline Normal sleep & $\begin{array}{l}\text { Cardiac parasympathetic predominance [24, 27]; } \\
\text { a slight increase in systolic BP in deep sleep } \\
\text { (NREM 3-4) [12, 24, 64, 65] }\end{array}$ & $\begin{array}{l}\text { Peripheral sympathetic activation [24], increased BP, heart rate } \\
\text { variability and BRS [12, 44, 64, 66-68]; } \\
\text { baroreflex control is more effective in buffering the increased } \\
\text { sympathetic activation at the end of the night sleep [69] }\end{array}$ \\
\hline OSA & $\begin{array}{l}\text { Disrupted nocturnal baroreflex gain and thus } \\
\text { increased chance of arrhythmic-ischemic events [24] }\end{array}$ & Alteration of BRS repatterning [14] \\
\hline
\end{tabular}

diac modulation, and one could divide them into the three groups of sleep phases: circadian (day-time wakefulness vs. night-time sleep), intraphasic (difference within NREM sleep stages) and phasic (difference between REM and NREM phases; table 1; fig. 1).

The autonomic network normally gives respiration a sleep-dependent repatterning (remarkably regular breathing in NREM sleep, but erratic in REM sleep [40]). Apneic events during REM sleep are associated with alteration of BRS phasic repatterning [14]. Disrupted nocturnal baroreflex gain during cyclic alternating patterns (micro-arousals) in NREM sleep may increase the chance of arrhythmic-ischemic events [24]. Nocturnal BRS is normally at its highest during deep sleep, but in OSA combined with EDS, it is found to be decreased [14].
Lombardi et al. [14] (2008) concluded that aggravated reduction of overnight BRS and increased sympatho-vagal balance in NREM sleep were positively associated with EDS.

As described above, sleep structure is normally associated with central autonomic regulation, with the specific behavior of cardiovascular reflexes modulating arterial BP (slight reduction or increase, changes of total variability) according to the sleep phase [12]. Within the first 3 days after onset of acute stroke, sleep structure is not only impaired but appears in a certain manner [30]. Thus, linked to the sleep structure, autonomic repatterning is also supposed to be impaired in stroke. As sleep fragmentation is linked to stroke severity [30], and NREM is the most prognostic sleep stage in stroke out- 
Fig. 2. Summarized autonomic findings in sleep, which probably play a major role in stroke development.

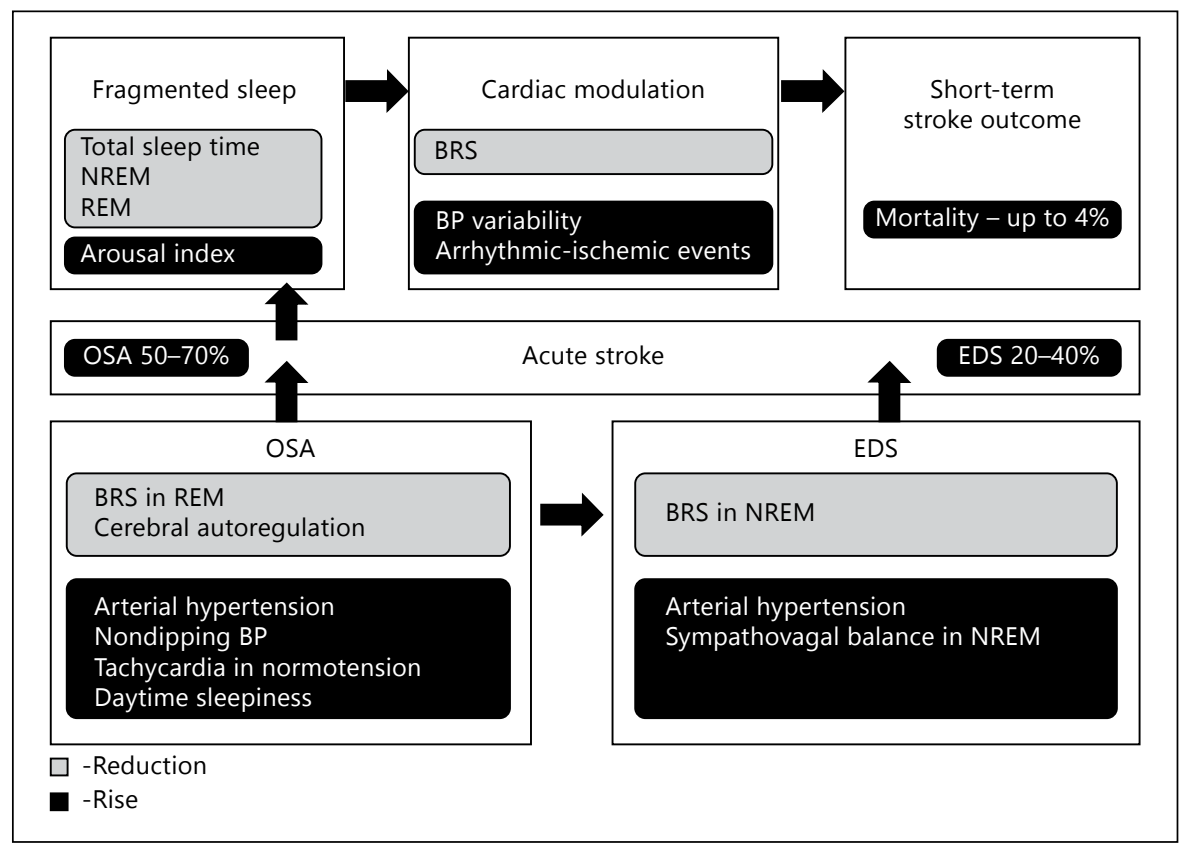

come $[9,10]$, impaired autonomic repatterning may relate to poststroke cardiovascular dysfunction. Moreover, better-illuminated pathogenesis of OSA may be important for understanding the mechanisms of stroke deterioration in sleep. The insula may be implicated in generating cardiac instability, resulting in more frequent arrhythmias in stroke patients [3]; as yet, no study has been undertaken to investigate systematically acute stroke development (fig. 2).

\section{Discussion}

Occurrence of poststroke early deterioration was estimated to be from 13 to $38 \%$ in different studies [48]. Recent statistics are lesser by half compared to those of the past decade and possibly due to the impact of modern treatments and stroke unit care [49]. It is hard to rely on even modern studies due to different definitions used for worsening detection. The majority of studies have several limitations compromising the accuracy of ANS findings in acute stroke due to lack of strict randomization (population, comorbid status, ongoing treatment, imprecise stroke locations/volume, etc.). Different techniques/parameters of ANS evaluation in stroke have been used, though in the normal population the reliability of nocturnal autonomic changes and sleep patterns are not established as well. Unfortunately, there has been no evalua- tion of the relationship between sleep architecture and ANS impairment in the first $72 \mathrm{~h}$ after stroke.

While our hypothesis is focused on ANS dysfunction during night sleep, it is sensitive to stroke localization. At the same time, poststroke sympathetic overactivity may reflect a general failure of regulation of cardiovascular functions (arrhythmia and BP variability) due to declining BRS or secondary brain injury worsening the infarction outcome [7]. In addition, stroke volume by itself positively correlates with NIHSS regardless of localization. We would suggest that dysfunction of both the ANS and central nervous system structures mediating arousal will correlate with more severe NIHSS, particularly the prominent sleep-regulating structures such as 'noradrenergic' pons (locus coeruleus) and 'cholinergic' thalamus (projections from pedunculopontine and laterodorsal tegmental nuclei [50]). Lesions of cerebrocortical afferents of the arousal system ('melanin-, orexin-, hypocretinergic' hypothalamus and 'acethylcholine-, GABAergic' basal forebrain) [50] may result in increased stroke severity due to circadian rhythm impairment. Insula, as a cerebral site of multifunctional integration, may also be a key point of sleep architecture, leading to BRS impairment, arrhythmias and deterioration in acute stroke.

Sleep-related hemodynamic changes were studied as a potential cause of nocturnal poststroke deterioration as well, but rather controversial data were obtained: the de- 
cline in MCA blood flow was associated only with the duration of obstructive hypopneas but not with OSA, and correlated with the magnitude of fall in arterial oxygen saturation from baseline [51]; changes of cerebral oxygenation saturation were more profound in the unaffected hemisphere, except for oxygenated hemoglobin, than in the affected hemisphere [52]. Impaired cerebral autoregulation during wakefulness was correlated with OSA severity [53], while severe disturbances of cerebrovascular reactivity and increase of arterial stiffness in MCA are more profound during consecutive respiratory events [51].

Even though early complex sleep and autonomic evaluation may be difficult, determining pathological sleep patterns and autonomic changes during the early stages of acute stroke could alert the physician to the potential for clinical deterioration. By being aware when deterioration occurs, it may be possible to intervene either with nonpharmacological or pharmacological means by treatment of acute autonomic dysfunction (i.e. altered BP mediated by autonomic dysfunction). Unfortunately, up to now there are no evidence-based therapeutic options available highlighting the necessity for further investigation.

Hypothetically, management of sleep-modulated autonomic imbalance may correspond to brain structures involved including pontine, thalamic or cortical/insular involvement. Pontine involvement with significant REM suppression $[29,54]$ is accompanied by suppression of the noradrenaline system leading to sudden death [55]. It was theorized that arousals in pontine lesions may be a protective physiological mechanism against sudden death [17], assuming that noradrenergic pontine structures (including the locus coeruleus) are most active in awake states after stroke [56]. This might be a potential reason to avoid CPAP in acute pontine stroke as OSA in this case is a secondary preventive mechanism. Moreover, it might explain why CPAP does not appear to improve calculated vascular risk [57].

In contrast, thalamic lesions in stroke are characterized by 'suspending' in the drowsy state (extended NREM $1)$, decreased arousal index and no major REM sleep alterations [58], supposedly accompanied by exaggerated sympathetic activation with preserved parasympathetic drive to the cardiovascular system [59]. The possible approach here could be controlling nondipping BP at bedtime (chronotherapy $[60,61]$ ) with angiotensin receptor blockers [62, 63].

Due to cerebral multifunctional association, insular lesions could be similar to cortical lesions (decreased
NREM 2-4 and REM but increased arousal index $[9,10$, 29-31]), but with more severe failure of NREM-associated nocturnal BRS gain and arrhythmias. Thus, CPAP would be an appropriate strategy to reduce arrhythmicischemic events.

\section{Conclusion}

Polysomnographic studies in acute stroke show sleep EEG changes associated with autonomic regulation of the cardiovascular system. Altered sleep architecture in stroke correlates with stroke severity and outcome. Nondipping arterial $\mathrm{BP}$, impaired nocturnal heart rate variability and BRS in OSA are linked to stroke outcome. Understanding of heart-brain interactions in sleep may have important implications for preventing stroke worsening and sudden death. We hypothesize that modification of sleep stages may improve outcome in patients with acute ischemic stroke. This may be necessary within 3 days after stroke onset, when EEG abnormalities show progressive poststroke deterioration that is linked to acute cardiovascular events including sudden death. Thus, early complex sleep and autonomic evaluation is a potential new strategy in stroke to improve clinical outcomes.

\section{Acknowledgment}

The authors would like to thank Dr. Colleen Ives for her critical appraisal of this manuscript.

\section{Disclosure Statement}

The authors have no conflicts of interest to disclose.

References

Cerebrovasc Dis 2013;36:454-461

1 Dávalos A, Castillo J: Progressing stroke; in Current Review of Cerebrovascular Disease. Philadelphia, Current Medicine Inc, 1999, pp 149-160.

2 Mackay J, Mensah G (eds): The Atlas of Heart Disease and Stroke. World Health Organization, 2004.

-3 Sörös P, Hachinski V: Cardiovascular and neurological causes of sudden death after ischaemic stroke. Lancet Neurol 2012;11: 179-188.

4 Thanvi B, Treadwell S, Robinson T: Early neurological deterioration in acute ischaemic stroke: predictors, mechanisms and management. Postgrad Med J 2008;84:412417. 
5 Dávalos A, et al: Neurological deterioration in acute ischemic stroke: potential predictors and associated factors in the European Cooperative Acute Stroke Study (ECASS) I. Stroke 1999;30:2631-2636.

6 Sykora M, et al: Baroreflex: a new therapeutic target in human stroke? Stroke 2009;40:e678e682.

7 Hilz MJ, et al: High NIHSS values predict impairment of cardiovascular autonomic control. Stroke 2011;42:1528-1533.

8 Yaggi HK, et al: Obstructive sleep apnea as a risk factor for stroke and death. N Engl J Med 2005;353:2034-2041.

-9 Bassetti CL, Aldrich MS: Sleep electroencephalogram changes in acute hemispheric stroke. Sleep Med 2001;2:185-194.

10 Harbison J, et al: Sleep-disordered breathing following acute stroke. QJM 2002;95:741-747.

11 Dütsch M, et al: Cardiovascular autonomic function in poststroke patients. Neurology 2007;69:2249-2255.

-12 Silvani A: Physiological sleep-dependent changes in arterial blood pressure: central autonomic commands and baroreflex control. Clin Exp Pharmacol Physiol 2008;35:987994.

13 Tank J, et al: Relationship between blood pressure, sleep K-complexes, and muscle sympathetic nerve activity in humans. Am J Physiol Regul Integr Comp Physiol 2003; 285:R208-R214.

$\checkmark 14$ Lombardi C, et al: Daytime sleepiness and neural cardiac modulation in sleep-related breathing disorders. J Sleep Res 2008;17:263270.

15 Carrington MJ, et al: Changes in cardiovascular function during the sleep onset period in young adults. J Appl Physiol 2005;98:468-476.

16 Nieto FJ, et al: Association of sleep-disordered breathing, sleep apnea, and hypertension in a large community-based study. Sleep Heart Health Study. JAMA 2000;283:18291836.

17 Ding J, et al: Sleep-disordered breathing and white matter disease in the brainstem in older adults. Sleep 2004;27:474-479.

18 Pasic Z, et al: Incidence and types of sleep disorders in patients with stroke. Med Arh 2011 65:225-227.

19 Hermann DM, Bassetti CL: Sleep-related breathing and sleep-wake disturbances in ischemic stroke. Neurology 2009;73:1313-1322.

-20 Carskadon MA, Rechtschaffen A: Monitoring and staging human sleep; in Kryger MK, Roth T, Dement WC (eds): Principles and Practice of Sleep Medicine. Philadelphia, Elsevier, 2005, pp 1359-1377.

21 Parrino L, et al: Cyclic alternating pattern (CAP) in normal sleep: polysomnographic parameters in different age groups. Electroencephalogr Clin Neurophysiol 1998;107:439450.

22 EEG arousals: scoring rules and examples: a preliminary report from the Sleep Disorders Atlas Task Force of the American Sleep Disorders Association. Sleep 1992;15:173-184.
3 Achermann P, Borbély AA: Coherence analysis of the human sleep electroencephalogram. Neuroscience 1998;85:1195-1208.

24 Iellamo F, et al: Baroreflex buffering of sympathetic activation during sleep: evidence from autonomic assessment of sleep macroarchitecture and microarchitecture. Hypertension 2004;43:814-819.

25 Patil SP: Technical aspects of sleep testing; in ACCP Sleep Medicine Board Review. Northbrook, American College of Chest Physicians, 2009, pp 19-26.

26 Hachinski VC, Mamelak M, Norris JW: Prognostic value of sleep morphology in cerebral infarction; in Lechner $\mathrm{H}$, Meyer J, Reivich M (eds): Cerebral Vascular Disease. Amsterdam, Excerpta Medica, 1977, pp 287292.

27 Hachinski VC, Mamelak M, Norris JW: Sleep morphology and prognosis in acute cerebrovascular lesions; in Lechner $\mathrm{H}$, Meyer J, Reivich M (eds): Cerebral Vascular Disease. Amsterdam, Excerpta Medica, 1977, pp 69-71.

28 Hachinski VC, Mamelak M, Norris JW: Clinical recovery and sleep architecture degradation. Can J Neurol Sci 1990;17:332335.

29 Giubilei F, et al: Sleep patterns in acute ischemic stroke. Acta Neurol Scand 1992;86:567571.

30 Müller C, et al: Visual and spectral analysis of sleep EEG in acute hemispheric stroke. Eur Neurol 2002;48:164-171

-31 Gottselig JM, Bassetti CL, Achermann P: Power and coherence of sleep spindle frequency activity following hemispheric stroke. Brain 2002;125(part 2):373-383.

32 Urakami Y: Relationships between sleep spindles and activities of the cerebral cortex after hemispheric stroke as determined by simultaneous EEG and MEG recordings. J Clin Neurophysiol 2009;26:248-256.

33 La Rovere MT, et al: Baroreflex sensitivity and heart-rate variability in prediction of total cardiac mortality after myocardial infarction. ATRAMI (Autonomic Tone and Reflexes after Myocardial Infarction) Investigators. Lancet 1998;351:478-484.

34 Davies RJ, et al: Non-invasive beat to beat arterial blood pressure during non-REM sleep in obstructive sleep apnoea and snoring. Tho$\operatorname{rax} 1994 ; 49: 335-339$.

35 Selic C, et al: Blood pressure evolution after acute ischemic stroke in patients with and without sleep apnea. Stroke 2005;36:26142618.

36 Britton M, Carlsson A, de Faire U: Blood pressure course in patients with acute stroke and matched controls. Stroke 1986;17:861864.

37 Robinson TG, et al: Cardiac baroreceptor sensitivity predicts long-term outcome after acute ischemic stroke. Stroke 2003;34:705712.

38 La Rovere MT, Maestri R, Pinna GD: Baroreflex sensitivity assessment - latest advances and strategies. Eur Cardiol 2011;7:89-92.
39 Cortelli P, et al: Cardiovascular autonomic dysfunction in normotensive awake subjects with obstructive sleep apnoea syndrome. Clin Auton Res 1994;4:57-62.

40 Cortelli P, Provini F: Sleep apnea, autonomic dysfunction, and vascular diseases; in Culebras A (ed): Sleep, Stroke and Cardiovascular Disease. Cambridge, Cambridge University Press, 2012, pp 33-47.

41 Schrezenmaier C, et al: Adrenergic and vagal baroreflex sensitivity in autonomic failure. Arch Neurol 2007;64:381-386.

42 Oppenheimer SM, et al: Cardiovascular effects of human insular cortex stimulation. Neurology 1992;42:1727-1732.

43 Abboud $\mathrm{H}$, et al: Insular involvement in brain infarction increases risk for cardiac arrhythmia and death. Ann Neurol 2006;59:691699.

44 Smyth HS, Sleight P, Pickering GW: Reflex regulation of arterial pressure during sleep in man. A quantitative method of assessing baroreflex sensitivity. Circ Res 1969;24:109121

45 Low PA, Benarroch EE (eds): Clinical Autonomic Disorders. Philadelphia, Lippincott Williams \& Wilkins, 2008, p 780.

46 Rudas L, et al: Human sympathetic and vagal baroreflex responses to sequential nitroprusside and phenylephrine. Am J Physiol 1999; 276:H1691-H1698.

47 Hart EC, et al: Baroreflex control of muscle sympathetic nerve activity: a nonpharmacological measure of baroreflex sensitivity. Am J Physiol Heart Circ Physiol 2010;298:H816H822.

48 Tsivgoulis G, et al: Hemodynamic causes of deterioration in acute ischemic stroke. Perspect Med 2012;1:177-184.

49 Norris JW, Hachinski V: The evolution and impact of acute stroke units on outcome in stroke patients. Can J Neurol Sci 2009;36: 274-276.

50 Schwartz JR, Roth T: Neurophysiology of sleep and wakefulness: basic science and clinical implications. Curr Neuropharmacol 2008;6:367-378

51 Netzer N, et al: Blood flow of the middle cerebral artery with sleep-disordered breathing: correlation with obstructive hypopneas. Stroke 1998;29:87-93.

52 Pizza F, et al: Cerebral hemodynamic changes in stroke during sleep-disordered breathing. Stroke 2012;43:1951-1953.

53 Furtner M, et al: Cerebral vasoreactivity decreases overnight in severe obstructive sleep apnea syndrome: a study of cerebral hemodynamics. Sleep Med 2009;10:875-881.

54 Hermida RC, et al: Relationship between metabolic syndrome, circadian treatment time, and blood pressure non-dipping profile in essential hypertension. Chronobiol Int 2011;28: 509-519.

55 Almirall J, et al: Effects of chronotherapy on blood pressure control in non-dipper patients with refractory hypertension. Nephrol Dial Transplant 2012;27:1855-1859. 


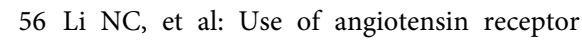
blockers and risk of dementia in a predominantly male population: prospective cohort analysis. BMJ 2010;340:b5465.

57 Rakugi H, et al: Comparison of the efficacy and safety of azilsartan with that of candesartan cilexetil in Japanese patients with grade I-II essential hypertension: a randomized, double-blind clinical study. Hypertens Res 2012;35:552-558.

58 Tosato $M$, et al: Sleep disruption following paramedian pontine stroke. BMJ Case Report 2009, Doi: 10.1136/bcr.07.2008.0460.

59 Lavezzi AM, Alfonsi G, Matturri L: Pathophysiology of the human locus coeruleus complex in fetal/neonatal sudden unexplained death. Neurol Res 2013;35:44-53.

60 Kalia M: Neurobiology of sleep. Metabolism 2006;55(suppl 2):S2-S6.
61 Craig SE, et al: Continuous positive airway pressure improves sleepiness but not calculated vascular risk in patients with minimally symptomatic obstructive sleep apnoea: the MOSAIC randomised controlled trial. Tho$\operatorname{rax} 2012 ; 67: 1090-1096$.

62 Bassetti C, et al: Hypersomnia following paramedian thalamic stroke: a report of 12 patients. Ann Neurol 1996;39:471-480.

63 Benarroch EE, Stotz-Potter EH: Dysautonomia in fatal familial insomnia as an indicator of the potential role of the thalamus in autonomic control. Brain Pathol 1998;8:527-530.

64 Somers VK, et al: Sympathetic nerve activity during sleep in normal subjects. N Engl J Med 1993;328:303-307.
65 Bristow JD, et al: Cardiovascular and respiratory changes during sleep in normal and hypertensive subjects. Cardiovasc Res 1969;3: 476-485.

66 Coccagna G, et al: Laboratory note. Arterial pressure changes during spontaneous sleep in man. Electroencephalogr Clin Neurophysiol 1971;31:277-281.

67 Davies RJ, et al: Arterial blood pressure responses to graded transient arousal from sleep in normal humans. J Appl Physiol 1993; 74:1123-1130.

68 Snyder F, et al: Changes in respiration, heart rate, and systolic blood pressure in human sleep. J Appl Physiol 1964;19:417-422.

69 Legramante JM, et al: Sleep-related changes in baroreflex sensitivity and cardiovascular autonomic modulation. J Hypertens 2003;21: 1555-1561.

\section{Erratum}

In the article by Kaste et al., entitled 'Safety, tolerability and pharmacokinetics of MCI186 in patients with acute ischemic stroke: new formulation and dosing regimen' [Cerebrovasc Dis 2013;36:196-204, DOI: 10.1159/000353680], the following information was erroneously omitted:

\section{Steering Committee}

Markku Kaste, Turgut Tatlisumak, Helsinki University Central Hospital, Finland; Peter Koudstaal, Diederik Dippel, Erasmus Medical Center, Rotterdam, The Netherlands; Gary Ford, Newcastle University, UK.

\section{Data and Safety Monitoring Committee}

Kennedy Lees, Western Infirmary, Glasgow, UK; Philip Bath, University of Nottingham, UK.

\section{Investigators and Institutions}

Finland: Turgut Tatlisumak; Risto O. Roine, Turku University Central Hospital. The Netherlands: Diederik Dippel; Koos Keizer, Catharina Ziekenhuis, Eindhoven; Tobien Schreuder, Atrium Medical Center, Heerlen. UK: Gary Ford, Anand Dixit, Royal Victoria Infirmary, Newcastle; Philippa Tyrrell, Salford Royal Hospital; Matthew Walters; Western Infirmary, Glasgow; Keith Muir, Southern General Hospital, Glasgow.

Nocturnal Deterioration after Ischemic Stroke and Autonomic Dysfunction 